\title{
Nailfold capillaroscopy in rheumatology: ready for the daily use but with care in terminology
}

\author{
Maurizio Cutolo ${ }^{1}$ - Sabrina Paolino ${ }^{1}$ - Vanessa Smith ${ }^{2,3,4}$
}

Received: 24 July 2019 / Accepted: 24 July 2019 / Published online: 8 August 2019

(C) International League of Associations for Rheumatology (ILAR) 2019

\section{The scleroderma pattern in systemic sclerosis}

With the recent inclusion of nailfold capillaroscopy (NVC) in the 2013 ACR/EULAR classification criteria for systemic sclerosis (SSc), together with the assessed role in monitoring the progression of the disease and effects of treatments, its use and the need to learn the correct interpretation and representation in daily practice, as well as in research context by rheumatologists, is increasing worldwide $[1,2]$.

In rheumatology today, NVC is the mandatory tool for the evaluation of subjects affected by Raynaud's phenomenon (RP) because a "scleroderma pattern" has been validated and helps to differentiate primary from secondary RP associated with connective tissue diseases (CTDS), in primis SSc [2-5].

As a matter of fact, microvascular changes, characterised by progressive structural and functional damage of the microvessels, play a central role in the complex pathogenesis of autoimmune diseases and in particular in SSc [6, 7].

The first modern interpretation of the microvascular alterations in SSc was given by Maricq et al. in 1980, using the wide-field microscopy technique, describing it as a capillaroscopic pattern [8].

Maurizio Cutolo

mcutolo@unige.it

Sabrina Paolino

sabrina.paolino@unige.it

1 Research Laboratories and Academic Division of Rheumatology, Department of Internal Medicine - IRCCS San Martino Polyclinic Hospital, University of Genova, Viale Benedetto XV, 6, 16132 Genoa, Italy

2 Department of Rheumatology, Ghent University Hospital, Ghent, Belgium

3 Department of Internal Medicine, Ghent University, Ghent, Belgium

4 Unit for Molecular Immunology and Inflammation, VIB Inflammation Research Center (IRC), Ghent, Belgium
This classification was later adapted to the videocapillaroscopic technique in 2000 by Cutolo et al., introducing a new concept on the basis of the microvascular morphological characteristics associated with disease progression [9-12].

The microvascular lesions detected qualitatively by NVC in SSc patients follow three clearly distinct patterns that differ from the normal aspect and reflect various phases of capillary involvement with an evolutive trend from the "Early", to the "Active", until the "Late" [13-16].

The different SSc NVC patterns correlate with the disease duration and severity, as well as the associated clinical complications and autoantibody profile [17-19].

In addition, the NVC patterns have been found to change in patients with SSc with up to 4 years of combined therapy, in particular showing a progressive significant recovery in structure as well as function of microvasculature, linked to improved clinical outcomes, independent of disease severity [20-22].

Furthermore, reliability of the qualitative and semiquantitative NVC assessment in SSc cohorts between raters at different centres has been demonstrated [23]. Reliability of NVC assessment is essential for the use of this tool in multicentre SSc trials.

The quantitative analysis of the morphological changes observed at NVC today is widely validated and even automatic systems for the count of capillaries are available [24-27].

\section{The scleroderma-like pattern in autoimmune connective tissues diseases}

Other NVC patterns have been searched in other CTDs, and a NVC "scleroderma-like "pattern that consists of a cluster of alterations of the capillary distribution, shape, number, and dimension pattern (mixing together the aspects observed in detail in the three SSc NVC patterns) has been described in dermatomyositis (DM) and recently compared on the long-term followup to the SSc NVC pattern changes (DM) [28-30]. 
Other studies described the association between microvessel array and inflammatory myositis (IIM), and often considering together DM and PM [31,32].

Among the almost 80 papers reported in the literature concerning capillaroscopy and DM and evaluating different aspects and interactions, the most recent investigation published by Miossi et al. reported several correlations between morphological markers of the NVC "scleroderma-like" pattern and circulating angiogenetic factors $(\mathrm{AF})$ in recent-onset DM [33].

The conclusion was that the increase of the serum AF angiogenin (ANG) in DM might represent a tool for the assessment of the role the angiogenesis process in DM which seems plausible with the very disturbed microvessel array observed in the "scleroderma-like" pattern.

The presence of capillaroscopic changes mixing the different NVC SSc pattern features (as described in the "sclerodermalike" pattern) was reported in another very recent investigation by Shenavandeh et al. in $33 \mathrm{DM}$ patients (58.7\%), and compared with the NVC patterns of $27 \mathrm{SSc}$ patients (79.4\%) as well as other CTDs, like mixed connective tissue disease (MCTD) and undifferentiated connective tissue disease (UCTD); however, possible overlaps were referred by the authors [34].

Of note, for the last two diseases (MCTD and UCTD), the number of patients analysed was not significant to draw any conclusion ( 3 and 2 patients, respectively).

The altered capillaroscopic findings when tested versus clinical aspects associated with CTDs, like the "Mechanic's Hands" (Gottron's papules), were found significantly correlated in $80.8 \%$ of CTD patients, including the concomitant presence of the RP.

Interestingly, in a previous study, the effect of treatment on capillaroscopic pattern in patients with DM showed that a rapid change of the NVC microvascular status during the time and treatment [35].

On the other hand, a recent multicentre investigation by Sebastiani et al. showed that NVC specific abnormalities are commonly observed in Antisynthetase Syndrome (AS), independently from the occurrence of RP [36].

Again, the presence of a NVC "scleroderma-like" pattern could allow the identification of a more defined AS subtype, possibly in the presence of inflammatory myopathy as a major clinical feature.

Interestingly, several studies analysed the NVC in systemic lupus erythematosus (SLE) [37].

Forty such studies were identified and displayed through a standardised language, more specifically the standard interpretation of capillaroscopic descriptions as consensed by the EULAR study group on microcirculation in rheumatic diseases (EULAR SG-MC see below).

When displaying the studies through a standardised representation language agreed by the EULAR SG-MC in rheumatic diseases, several NVC parameters were found to be significantly more prevalent in SLE patients when compared with healthy controls, such as tortuous capillaries, abnormal morphology and haemorrhages, whereas hairpin-shaped capillaries were found to be significantly less prevalent than in healthy persons.

The semi-quantitatively determined nailfold capillaroscopic score (NFC score) was also found higher in SLE patients. Some correlations between clinical parameters, like disease activity and NVC parameters (abnormal morphology, i.e. "meandering"), and NFC score were found in seven studies and in one study with haemorrhages. Frequent episodes of RP and gangrene were found significantly correlated with dilated capillaries. Conflicting results were found about other immune parameters.

On the other hand, "scleroderma-like" capillaroscopic changes can also be found in rheumatoid arthritis (RA) patients, with peripheral digital vasculitis, as recently summarised in a short article by Lambova et al. [38].

Interestingly, the authors conclude that these changes are not obligatory but possibly associated with an overlap syndrome. Further larger studies are required.

Very recently, several noninfectious vasculitides have been analysed by a systematic review by Bertolazzi et al. starting from the concept that vasculitis characterised by active involvement of vessels including capillaries might offer a large field of investigation for the use of NVC [39].

The vasculitides included Takayasu arteritis (TAO), giant cell arteritis, polyarteritis nodosa, Kawasaki disease, ANCAassociated vasculitis, microscopic polyangiitis, granulomatosis with polyangiitis, eosinophilic granulomatosis (GPA), crioglobulinemic vasculitis (MC), IgA vasculitis (IAV), Behçet disease (BD), Cogan's syndrome, lupus vasculitis, rheumatoid vasculitis and sarcoid vasculitis.

The authors did not explain in their methodologic section the definitions that had been used throughout the studies; hence, interpretability of the findings of this systematic review is not without sine cure. This is because same terminology may have different meaning by different authors.

Also, contrary to the systematic review on SLE (above), the authors of this manuscript did not use an upfront standard language (see below) to display the results of the manuscripts they had identified. This may be the reason that they found "a wide range of NVC changes" and inconclusive evidence for clinical impact of capillaroscopy.

We agree with the authors that future large-scale standardised studies are required to elucidate on the role of capillaroscopy in vasculitis.

\section{The standardisation of the terminology in nailfold capillaroscopy}

Since one of the major problems in analysing and reporting the morphological changes observed by NVC in 
rheumatology is related to the need of a standardisation of the terminology, two consecutive recent studies reported on the reliability of simple capillaroscopic definitions in describing capillary morphology in rheumatic diseases [40, 41].

In these studies by the EULAR SG-MC in rheumatic diseases, a simple definition was proposed to describe the morphology of single capillaries as being normal or abnormal. A moderate reliability regarding these definitions was obtained between attendees of the sixth EULAR capillaroscopy course, held in 2014, and an excellent reliability after optimatisation of the definition, at the seventh EULAR capillaroscopy course held in 2016 in Genoa (Italy).

Attendees were rheumatologists with different levels of expertise in performing capillaroscopy (a large number of whom had no previous experience). The multicentre international study (37 countries) demonstrated that the reliability of the optimised simple capillaroscopic definition of normal and abnormal morphologies of capillaries is excellent, with a mean proportion of overall agreement of $91 \%$, and a mean proportion of specific agreement for abnormal of $89 \%$ and for normal of $92 \%$, even when used by rheumatologists with different levels of expertise in capillaroscopy.

This standardisation of evaluation of morphology of single capillaroscopic shapes has now been followed by a multicentre consensed "fast track algorithm" by the EULAR SG-MC in rheumatic diseases [42].

This latter study not only presented the EULAR consensed standard language to describe overall capillaroscopy and existing capillaroscopic manuscript in a standardised, uniform way (number of capillaries [density], dimension, abnormal shapes [morphology], presence/absence of haemorrhages) but also described for the first time an algorithm to discern scleroderma patterns from non-scleroderma patterns in a simple way and hence allowed novices as well as expert capillaroscopists to have a simple algorithm to discern scleroderma patterns form non-scleroderma patterns, as well as the golden standard.

This implies that the key role of capillaroscopy, more specifically to discern a scleroderma pattern from a nonscleroderma with the aim to distinguish a primary RP from a secondary RP due to SSc, is now made easy for a capillaroscopist of any level.

A more recent review article by Karbalaie et al. was addressed to identify different factors affecting the reliability and validity of the assessment in NVC [43].

The investigation concluded that minimisation of the impact of some artefacts should be obtained just by considering dedicated protocols before the examination and by using training and guidelines, also with the intention to reduce mistakes in the measurement and analysis of NVC images.

Following the EULAR and ACR-dedicated courses and study groups on teaching basic, applied and practical capillaroscopy to the rheumatologists, a recent investigation on consensus for the format and content of the report in capillaroscopy in Rheumatology by the PANLAR Capillaroscopy Study Group has been published by Bertolazzi et al. [44].

A Delphi questionnaire was sent to a South American panel including 25 rheumatological experts in capillaroscopy, asking them to rate their level of agreement or disagreement with each statement. The exercise consisted of three online rounds and a face-to-face (live meeting) held at the PANLAR 2018 congress in Buenos Aires, Argentina. At the end of the exercise, 46 recommendations were formulated as further effort for the standardization of technique of execution and interpretation of NVC.

The results of the report confirmed the conclusions of the earliest studies previously mentioned [40,41], namely that the capillaroscopy examination and its use in a homogeneous form can help in the correct interpretation of findings in daily practice.

Against this background, the recent international consensus of worldwide experts to find a standard format to report capillaroscopic characteristics (density, dimension, abnormal shapes, haemorrhages) and to create an algorithm that makes interpretation of scleroderma pattern or not has been more than welcome and will be a working tool at an international level [42].

\section{Conclusions}

Finally, in order to further implement the already existing EULAR living courses and dedicated textbook started at the beginning of 2000s, the EULAR School of Rheumatology (ESoR) has now implemented preparations for an online course on Capillaroscopy and Microcirculation in Rheumatic Musculoskeletal Diseases (RMD).

In conclusion, nailfold capillaroscopy is an important, reliable and useful tool in rheumatology: it is ready for daily use but with care in interpretation and terminology.

\section{References}

1. Van den Hoogen F, Khanna D, Fransen J, Johnson SR, Baron M, Tyndall A et al (2013) 2013 classification criteria for systemic sclerosis: an American College of Rheumatology/European League against Rheumatism collaborative initiative. Arthritis Rheum 65: 2737-2747

2. Snow MH, Saketkoo LA, Frech TM, Stever JR, Lebedoff N, Herrick AL et al (2019) Results from an American pilot survey among Scleroderma Clinical Trials Consortium members on capillaroscopy use and how to best implement nailfold capillaroscopy training. Clin Exp Rheumatol

3. Koenig M, Joyal F, Fritzler MJ, Roussin A, Abrahamowicz M, Boire G, Goulet JR, Rich É, Grodzicky T, Raymond Y, Senécal 
JL (2008) Autoantibodies and microvascular damage are independent predictive factors for the progression of Raynaud's phenomenon to systemic sclerosis: a twenty-year prospective study of 586 patients, with validation of proposed criteria for early systemic sclerosis. Arthritis Rheum 58:3902-3912

4. Cutolo M, Sulli A, Smith V (2010) Assessing microvascular changes in systemic sclerosis diagnosis and management. Nat Rev Rheumatol 6:578-587

5. Ingegnoli F, Ughi N, Dinsdale G, Orenti A, Boracchi P, Allanore Y et al (2017) An international SUrvey on non-iNvaSive tecHniques to assess the mIcrocirculation in patients with RayNaud's phEnomenon (SUNSHINE survey). Rheumatol Int 37:1879-1890

6. Masi A, Bijlsma JWJ, Chikanza IC, Pitzalis C, Cutolo M (1999) Neuroendocrine, immunologic, and microvascular systems interactions in rheumatoid arthritis: physiopathogenetic and therapeutic perspectives. Sem Arthritis Rheum 29:65-81

7. Cutolo M, Soldano S, Smith V (2019) Pathophysiology of systemic sclerosis: current understanding and new insights. Expert Rev Clin Immunol 15:753-764

8. Maricq HR (1981) Wide-field capillary microscopy. Arthris Rheum 24:1159-1164

9. Cutolo M, Sulli A, Pizzorni C, Accardo S (2000) Nailfold videocapillaroscopy assessment of microvascular damage in systemic sclerosis. J Rheumatol 27:155-160

10. Smith V, Decuman S, Sulli A, Bonroy C, Piettte Y, Deschepper E, de Keyser F, Cutolo M (2012) Do worsening scleroderma capillaroscopic patterns predict future severe organ involvement? A pilot study. Ann Rheum Dis 71:1636-1639

11. Smith V, Riccieri V, Pizzorni C, Decuman S, Deschepper E, Bonroy C, Sulli A, Piette Y, de Keyser F, Cutolo M (2013) Nailfold capillaroscopy for prediction of novel future severe organ involvement in systemic sclerosis. J Rheumatol 40:2023-2028

12. Cutolo M, Herrick AL, Distler O, Becker MO, Beltran E, Carpentier P et al (2016) Nailfold videocapillaroscopic features and other clinical risk factors for digital ulcers in systemic sclerosis: a multicenter, prospective cohort study. Arthritis Rheumatol 68: 2527-2539

13. Cutolo M, Pizzorni C, Sulli A (2005) Capillaroscopy. Best Pract Res Clin Rheumatol 19:437-452

14. Herrick AL, Cutolo M (2010) Clinical implications from capillaroscopic analysis in patients with Raynaud's phenomenon and systemic sclerosis. Arthritis Rheum 62:2595-2604

15. Sulli A, Pizzorni C, Smith V, Zampogna G, Ravera F, Cutolo M (2012) Timing of transition between capillaroscopic patterns in systemic sclerosis. Arthritis Rheum 64:821-825

16. Cutolo M, Sulli A, Smith V (2013) How to perform and interpret capillaroscopy. Best Pract Res Clin Rheumatol 27:237-248

17. Cutolo M, Pizzorni C, Tuccio M, Burroni A, Craviotto C, Basso M et al (2004) Nailfold videocapillaroscopic patterns and serum autoantibodies in systemic sclerosis. Rheumatology 43:719-726

18. Caramaschi P, Canestrini S, Martinelli N, Volpe A, Pieropan S, Ferrari $M$ et al (2017) Scleroderma patients nailfold videocapillaroscopic patterns are associated with disease subsets and disease severity. Rheumatology 46:1566-1569

19. Ingegnoli F, Gualtierotti R (2013) A systematic overview on the use and relevance of capillaroscopy in systemic sclerosis. Exp Rev Clin Immunol 9:1091-1097

20. Cutolo M, Zampogna G, Vremis L, Smith V, Pizzorni C, Sulli A (2013) Longterm effects of endothelin receptor antagonism on microvascular damage evaluated by nailfold capillaroscopic analysis in systemic sclerosis. J Rheumatol 40:40-45

21. Cutolo M, Ruaro B, Pizzorni C, Ravera F, Smith V, Zampogna G, Paolino S, Seriolo B, Cimmino M, Sulli A (2014) Longterm treatment with endothelin receptor antagonist bosentan and iloprost improves fingertip blood perfusion in systemic sclerosis. J Rheumatol 41:881-886
22. Trombetta AC, Pizzorni C, Ruaro B, Paolino S, Sulli A, Smith V, Cutolo M (2016) Effects of longterm treatment with bosentan and iloprost on nailfold absolute capillary number, fingertip blood perfusion, and clinical status in systemic sclerosis. J Rheumatol 43: 2033-2041

23. Smith V, Pizzorni C, De Keyser F, Decuman S, Van Praet JT, Deschepper E et al (2010) Reliability of the qualitative and semiquantitative nailfold videocapillaroscopy assessment in a systemic sclerosis cohort: a two-centre study. Ann Rheum Dis 69:1092-1096

24. Berks M, Tresadern P, Dinsdale G, Murray A, Moore T, Herrick A et al (2014) An automated system for detecting and measuring nailfold capillaries. Med Image Comput Comput Assist Interv 17: 658-665

25. Dinsdale G, Moore T, O'Leary N, Berks M, Roberts C, Manning J, Allen J, Anderson M, Cutolo M, Hesselstrand R, Howell K, Pizzorni C, Smith V, Sulli A, Wildt M, Taylor C, Murray A, Herrick AL (2017) Quantitative outcome measures for systemic sclerosis-related Microangiopathy - reliability of image acquisition in Nailfold Capillaroscopy. Microvasc Res 113:56-59

26. Mihai C, Smith V, Dobrota R, Gheorghiu AM, Cutolo M, Distler O (2018) The emerging application of semi-quantitative and quantitative capillaroscopy in systemic sclerosis. Microvasc Res 118:113120

27. Cutolo M, Trombetta AC, Melsens K, Pizzorni C, Sulli A, Ruaro B, Paolino S, Deschepper E, Smith V (2018) Automated assessment of absolute nailfold capillary number on videocapillaroscopic images: proof of principle and validation in systemic sclerosis. Microcirculation 25:e12447

28. De Angelis R, Cutolo M, Gutierrez M, Bertolazzi C, Salaffi F, Grassi W (2012) Different microvascular involvement in dermatomyositis and systemic sclerosis. A preliminary study by a tight videocapillaroscopic assessment. Clin Exp Rheumatol 30:S67-S70

29. Manfredi A, Sebastiani M, Cassone G, Pipitone N, Giuggioli D, Colaci M, Salvarani C, Ferri C (2015) Nailfold capillaroscopic changes in dermatomyositis and polymyositis. Clin Rheumatol 34:279-284

30. Pizzorni C, Cutolo M, Sulli A, Ruaro B, Trombetta AC, Ferrari G, Pesce G, Smith V, Paolino S (2018) Long-term follow-up of nailfold videocapillaroscopic changes in dermatomyositis versus systemic sclerosis patients. Clin Rheumatol 37:2723-2729

31. Mercer LK, Moore TL, Chinoy H, Murray AK, Vail A, Cooper RG, Herrick AL (2010) Quantitative nailfold video capillaroscopy in patients with idiopathic inflammatory myopathy. Rheumatology (Oxford) 49:1699-1705

32. Selva-O'Callaghan A, Fonollosa-Pla V, Trallero-Araguás E, Martínez-Gómez X, Simeon-Aznar CP, Labrador-Horrillo M, Vilardell-Tarrés M (2010) Nailfold capillary microscopy in adults with inflammatory myopathy. Semin Arthritis Rheum 39:398-404

33. Miossi R, de Souza FHC, Shinjo SK (2019) Nailfold capillary changes in adult new-onset dermatomyositis: a prospective crosssectional study. Clin Rheumatol. https://doi.org/10.1007/s10067019-04537-x

34. Shenavandeh S, Habibi S, Habibi Y, Nazarinia M (2019) Mechanic hands: clinical and capillaroscopy manifestations of patients with connectivetissue diseases presented with and without mechanic hands. Clin Rheumatol. https://doi.org/10.1007/s10067-01804422-z

35. Mugii N, Hasegawa M, Matsushita T, Hamaguchi Y, Horie S, Yahata $\mathrm{T}$ et al (2011) Association between nail-fold capillary findings and disease activity in dermatomyositis. Rheumatology (Oxford) 50:1091-1098

36. Sebastiani M, Triantafyllias K, Manfredi A, González-Gay MA, Palmou-Fontana N et al (2019) Nailfold Capillaroscopy Characteristics of Antisynthetase Syndrome and Possible Clinical Associations: Results of a Multicenter International Study. 
American and European Network of Antisynthetase Syndrome Collaborative Group. J Rheumatol 46:279-284

37. Cutolo M, Melsens K, Wijnant S, Ingegnoli F, Thevissen K, De Keyser F et al (2018) Nailfold capillaroscopy in systemic lupus erythematosus: a systematic review and critical appraisal. Autoimmun Rev 17:344-352

38. Lambova SN, Müller-Ladner U (2019) Capillaroscopic features of microangiopathy in rheumatoid arthritis patients with peripheralvascular syndrome. Clin Rheumatol. https://doi.org/10. 1007/s10067-019-04561-x

39. Bertolazzi C, Gallegos-Nava S, Villarreal-Treviño AV, AlfaroRodriguez A, Clavijo-Cornejo D, Gutierrez M (2019) The current role of capillaroscopy in vasculitides. Clin Rheumatol. https://doi. org/10.1007/s10067-018-4399-1

40. Smith V, Beeckman S, Herrick AL, Decuman S, Deschepper E, De Keyser F et al (2016) An EULAR study group pilot study on reliability of simple capillaroscopic definitions to describe capillary morphology in rheumatic diseases. Rheumatology (Oxford) 55: 883-890

41. Cutolo M, Melsens K, Herrick AL, Foeldvari I, Deschepper E, De Keyser F et al (2018) Reliability of simple capillaroscopic definitions in describing capillary morphology in rheumatic diseases. Rheumatology (Oxford) 57:757-759

42. Smith V, Vanhaecke A, Herrick A, Distler O, Guerra M, Denton C, et al. (2019) Fast track algorithm: how to differentiate a "scleroderma pattern" from a "non-scleroderma pattern". Autoimmun Rev 2019. [In Press]

43. Karbalaie A, Emrani Z, Fatemi A, Etehadtavakol M, Erlandsson BE (2019) Practical issues in assessing nailfold capillaroscopic images: a summary. Clin Rheumatol. https://doi.org/10.1007/s10067-01904644-9

44. Bertolazzi C, Vargas Guerrero A, Rodríguez-Reyna TS, Sandoval H, Álvarez-Hernández E, Audisio MJ et al (2019) Pan-American League of Associations for Rheumatology (PANLAR) capillaroscopy study group consensus for the format and content of the report in capillaroscopy in rheumatology. Clin Rheumatol. https://doi.org/10.1007/s10067-019-04610-5

Publisher's note Springer Nature remains neutral with regard to jurisdictional claims in published maps and institutional affiliations. 